\title{
Thrombotic thrombocytopenic purpura and focal segmental glomerulosclerosis associated with the use of ecstasy
}

\author{
Yusuf Kayar, Nuket Bayram Kayar ${ }^{1}$, Venkatanarayana Gangarapu
}

Ecstasy is a drug, which causes serious side effects and sometimes it can be lethal. These effects are due to idiosyncratic reactions as a result of various stimulations in adrenergic receptors. Here we present a case of a 36-year-old male patient who was diagnosed with thrombotic thrombocytopenic purpura associated with the use of ecstasy. Plasmapheresis along with methylprednisolone treatment restores patient condition to normal.

Keywords: Ecstasy, glomerulosclerosis, thrombotic thrombocytopenic purpura

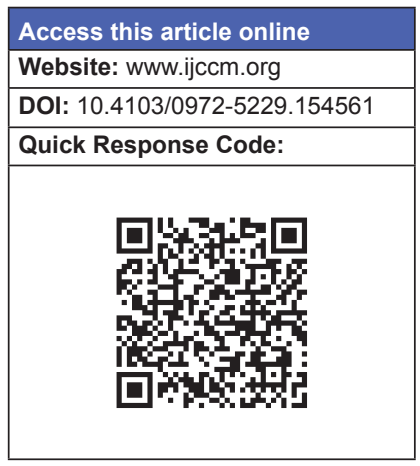

\section{Introduction}

While ecstasy is a drug used for fun today, it has serious side effects and sometimes it can be fatal. These effects are due to idiosyncratic reactions as a result of various stimulations in adrenergic receptors. ${ }^{[1]}$ Short after it is swallowed, it causes sweating, tachycardia, tiredness, muscle spasms, and jaw locking; and in long-term, it may cause delirium, convulsions, cerebral hemorrhage, cardiac arrhythmias, hyperthermia, liquid and electrolyte imbalance, disseminated intravascular coagulation (DIC), rhabdomyolysis, acute kidney failure, and acute liver failure. ${ }^{[1-4]}$ In this case report, we present a case of thrombotic thrombocytopenic purpura (TTP) associated with the use of ecstasy.

\section{Case Report}

This case is a 36-year-old male who complains of fever, speech difficulty, change in conscience, and red rashes

From:

Department of Internal Medicine, Bezmialem Vakif University, 'Department of Family Medicine, Bagcilar Education and Research Hospital, Istanbul, Turkey

Correspondence:

Dr. Yusuf Kayar, Department of Internal Medicine, Bezmialem Vakif

University, Vatan street, Fatih / Istanbul / Turkey 34093

E-mail: ykayar@yahoo.com on legs. Physical examination showed that the patient was drowsy, disoriented and uncooperative. In addition, he was pale, icteric and had petechial rashes on his legs. His blood pressure was $150 / 100 \mathrm{mmHg}$, and heart rate 92 beats/min in normal rhythm. His tests revealed that he had anemia and thrombocytopenia, increased lactate dehydrogenase $(\mathrm{LDH})$, indirect bilirubin and creatinine kinase, a two-fold increase in transaminases, and reticulocytes were $7.4 \%$. Direct and indirect Coombs tests were negative. His coagulation tests, D-dimer, fibrinogen, and creatinine were normal. His urinalysis revealed that he had proteinuria, calculated to be $320 \mathrm{mg} / 24-\mathrm{h}$. In his peripheral smear, there were $8-10$ schistocytes, $56 \%$ fractioned neutrophils, $22 \%$ clubbings, $14 \%$ lymphocytes, $4 \%$ monocytes, $2 \%$ myelocytes and $2 \%$ metamyelocytes in every region in the small magnification [Figure 1], so he was diagnosed as microangiopathic hemolytic anemia. Together with thrombocytopenia, fever, neurologic symptoms and proteinuria, the patient was labeled as TTP. His cranial tomography and cranial magnetic resonance imaging (MRI) did not reveal any pathology. The patient did not use any medication, did not have any autoimmune condition, and did not have any infection findings, and his abdominal and thorax tomography taken for any possible malignancy did not reveal any pathology. His viral serology 


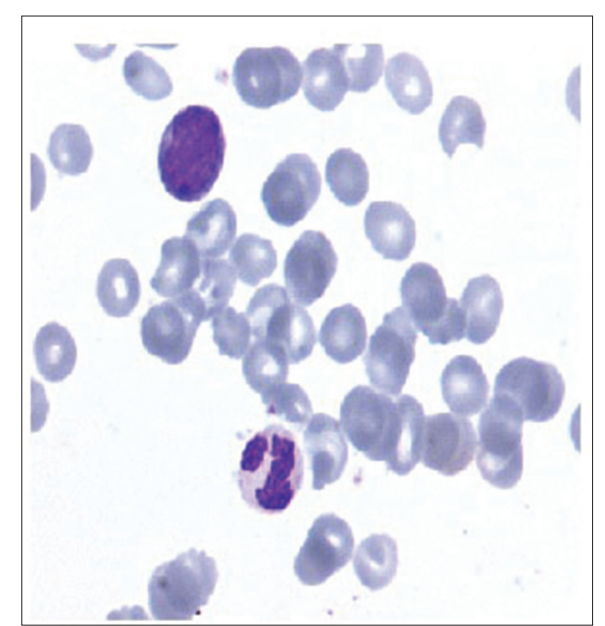

Figure I: In his peripheral smear, schistocytes in every region in the small magnification

tests and anti-nuclear antibodies, anti-neutrophil cytoplasmic antibodies, extractable nuclear antigens screen, rheumatoid factor, anti-cardiolipin $\operatorname{IgM}$, and IgG were negative. IgA, IgG, IgM, C3, C4 were found to be normal. As the patient had been using 3,4 methylenedioxymethamphetamine (MDMA) (ecstasy) intensively for approximately 10 years, his condition was diagnosed as TTP associated with ecstasy. Plasmapheresis was performed on the patient every day. He was given $1 \mathrm{~g} /$ day methylprednisolone and vincristine $2 \mathrm{mg}$ / day (single dose) for 3 days. When his platelets got above $100.000 \mathrm{~mm}^{3}$, plasmapheresis was started to be performed once every 2 days. The patient's LDH decreased, and level of his platelets increased so he was discharged. On follow-up visits he had left flank pain, decreased urine output and was hypertensive $(200 / 120 \mathrm{mmHg})$, so he was put on amlodipine $10 \mathrm{mg} /$ day and ramipril $5 \mathrm{mg} /$ day. Follow-up laboratory workup showed creatinine to be $2.4 \mathrm{mg} / \mathrm{dL}$, creatine kinase: $559 \mathrm{U} / \mathrm{L}$, platelet: $130.000 \mathrm{~mm}^{3}$, LDH: $351 \mathrm{U} / \mathrm{L}$ and $24-\mathrm{h}$ urine protein $7.6 \mathrm{~g} /$ day. Abdominal ultrasonography, MRI, and MRI angiography did not reveal any pathology. Kidney biopsy was performed and showed bowman capsules to have peak lesions in some glomeruli, along with adhesions and visceral epithelial proliferation. In these areas, focal minimal atrophy with few foamy cell group tubules, and a mild wall thickening in arterioles and arteries were observed. With these findings, the patient was diagnosed with focal segmental glomerulosclerosis (FSGS) [Figure 2]. It was thought that the patient developed FSGS secondary to ecstasy, and his proteinuria increased as a result of accelerated hypertension. The patient was given $1 \mathrm{~g} /$ day pulse methylprednisolone and cyclophosphamide for 3 days. Then, the patient's treatment continued with $1 \mathrm{mg} / \mathrm{kg}$ / day methylprednisolone, and his hypertension

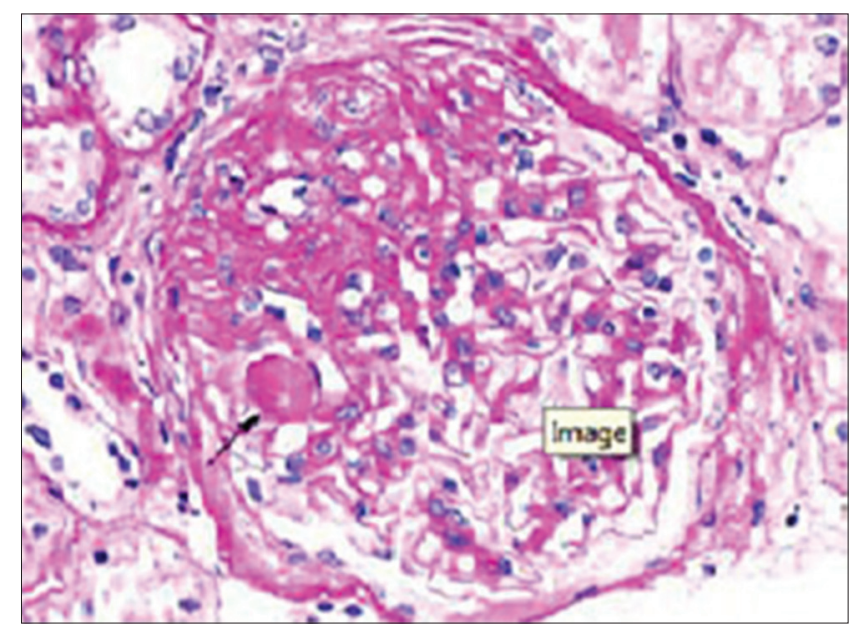

Figure 2: Focal minimal atrophy in a few foamy cell group tubules and a mild wall thickening in arterioles and arteries were observed

was controlled. Two weeks later, proteinuria lowered to $180 \mathrm{mg}$ / day. Plasmapheresis was performed on the patient for 1 more week, and when his LDH and platelets level turned to normal, his apheresis procedures were ended. The patient's methylprednisolone treatment dose was decreased, and he was discharged. Laboratory workup after 3 months showed levels of platelets, creatinine, creatine kinase and LDH to be normal.

\section{Discussion}

Thrombocytopenic purpura is a rare clinical syndrome with the cardinal features of hemolytic anemia, thrombocytopenia, fever, renal impairment, and neurological dysfunction, although all five features being observed in only $40 \%$ of the patients..$^{[5]}$ TTP is usually idiopathic, but it can be seen as a secondary condition after infections, medications, autoimmune diseases, malignancies, pregnancy, and bone marrow transplantation. ${ }^{[6]}$ While TTP was observed to develop after the use of cocaine and heroin, very rare cases were reported of TTP after the use of ecstasy. ${ }^{[7,8]}$ TTP was first defined in 1924 by Moschcowitz, and it occurs through immune and nonimmune mechanisms ${ }^{[6]}$ Recently, a specific metalloproteinase (ADAMTS13) that breaks up the von Willebrand factor was defined. Moderate or severe ADAMTS13 enzyme activity deficiency was seen in most TTP cases. ${ }^{[6]}$ While enzyme activity deficiency can be congenital, it has also been shown that it can be caused by auto-antibodies. ${ }^{[4]}$ With the absence of signs of any infection in our case, and the presence of hemolytic anemia, normal creatinine, proteinuria, fever, and change in consciousness, we diagnosed the patient as TTP rather than hemolytic uremic syndrome. With the use of amphetamine, vasoconstriction develops, followed by endothelial damage. Procoagulant factors are released from platelets, and microthrombuses are 
formed in arteriol capillary level, resulting in secondary microangiopathic hemolytic anemia. ${ }^{[7]}$ Three cases of TTP after consumption of amphetamines were described in the literature. ${ }^{[7-9]}$ We hypothesize that there could be an association between the consumption of MDMA in our case and the induction of TTP based on certain similarities between the molecular structure of MDMA and thienopyridines that are known to cause TTP.

The relationship between heroine and nephropathy has been known since $1970 .{ }^{[2]}$ Nephropathy due to cocaine occurs especially after the rhabdomyolysis. ${ }^{[3]}$ Nephropathy secondary to ecstasy use was first defined in 2007 by Bohatyrewicz et al. as a membranous glomerulonephritis that developed secondary to ecstasy use. ${ }^{[3]}$

Dehydration occurs secondary to hyperthermia after ecstasy use. Then hyponatremia occurs due to the antidiuretic hormone secreted as a response and drinking too much water. The rennin angiotensin aldosterone system is activated, and hence, water and salt are retained in the body. ${ }^{[4]}$ It exhibits sympathomimetic effect by stimulating adrenergic receptors, by blocking catecholamine reuptake and by inhibiting monoamine oxidase activity ${ }^{[4]}$ Hypertension occurs as a result of all these mechanisms. ${ }^{[4]}$ While chronic kidney failure can develop due to hypertension within weeks or months after ecstasy use, acute kidney failure and nephropathy can also develop as a result of accelerated hypertension. ${ }^{[4]}$ In our case, who developed nephropathy secondary to ecstasy use (FSGS), distinctive increase in proteinuria as a result of accelerated hypertension was observed.
In addition, it should be kept in mind that DIC, rhabdomyolysis, and ecstasy can have a direct toxic effect on nephropathy. As a result, although reported very rarely, it should be kept in mind that TTP accelerated hypertension and nephropathy can develop secondary to ecstasy use.

\section{References}

1. Henry JA, Jeffreys KJ, Dawling S. Toxicity and deaths from 3, 4-methylenedioxymethamphetamine ("ecstasy"). Lancet 1992;340:384-7.

2. Ginsberg MD, Hertzman M, Schmidt-Nowara WW. Amphetamine intoxication with coagulopathy, hyperthermia, and reversible renal failure. A syndrome resembling heatstroke. Ann Intern Med 1970;73:81-5.

3. Bohatyrewicz M, Urasinska E, Rozanski J, Ciechanowski K. Membranous glomerulonephritis may be associated with heavy marijuana abuse. Transplant Proc 2007;39:3054-6.

4. Woodrow G, Harnden P, Turney JH. Acute renal failure due to accelerated hypertension following ingestion of 3 , 4-methylenedioxymethamphetamine ('ecstasy'). Nephrol Dial Transplant 1995;10:399-400.

5. Murrin RJ, Murray JA. Thrombotic thrombocytopenic purpura: Aetiology, pathophysiology and treatment. Blood Rev 2006;20:51-60.

6. Qasim ZA, Partridge RA. Thrombotic thrombocytopenic purpura presenting as bilateral flank pain and hematuria: A case report. J Emerg' Med 2001;21:15-20.

7. Schirren CA, Berghaus TM, Sackmann M. Thrombotic thrombocytopenic purpura after Ecstasy-induced acute liver failure. Ann Intern Med 1999;130:163.

8. De Fallois J, Regenthal R, Petros S. Fulminant thrombotic thrombocytopenic purpura (TTP): Association with amphetamine consumption? Ann Hematol 2015;94:337-8.

9. Shibolet O, Kalish Y, Gillis S, Ilan Y. Hepatic and hematological complications following ecstasy usage. Harefuah 2001;140:911-4.

How to cite this article: Kayar Y, Kayar NB, Gangarapu V. Thrombotic thrombocytopenic purpura and focal segmental glomerulosclerosis associated with the use of ecstasy. Indian J Crit Care Med 2015;19:230-2.

Source of Support: Nil, Conflict of Interest: None declared. 